\title{
Identification of key genes in hepatocellular carcinoma and validation of the candidate gene, cdc25a, using gene set enrichment analysis, meta-analysis and cross-species comparison
}

\author{
XIAOXU LU ${ }^{1 *}$, WEN SUN $^{1 *}$, YANPING TANG ${ }^{1 *}$, LINGQUN ZHU $^{1}$, YUAN LI $^{1}, \mathrm{CHAO} \mathrm{OU}^{1}$, \\ CHUN YANG ${ }^{1}$, JIANJIA SU ${ }^{1}$, CHENGPIAO LUO ${ }^{1}$, YANLING $\mathrm{HU}^{2}$ and $\mathrm{JI} \mathrm{CAO}^{1}$ \\ ${ }^{1}$ Department of Research, Affiliated Tumor Hospital of Guangxi Medical University, Nanning, Guangxi 530021; \\ ${ }^{2}$ The Medical Scientific Research Center, Guangxi Medical University, Nanning, Guangxi 530022, P.R. China
}

Received December 11, 2014; Accepted October 26, 2015

DOI: $10.3892 / \mathrm{mmr} .2015 .4646$

\begin{abstract}
The aim of the present study was to determine key pathways and genes involved in the pathogenesis of hepatocellular carcinoma (HCC) through bioinformatic analyses of HCC microarray data based on cross-species comparison. Microarray data of gene expression in HCC in different species were analyzed using gene set enrichment analysis (GSEA) and meta-analysis. Reverse transcription-quantitative polymerase chain reaction and western blotting were performed to determine the mRNA and protein expression levels of cdc25a, one of the identified candidate genes, in human, rat and tree shrew samples. The cell cycle pathway had the largest overlap between the GSEA and meta-analysis. Meta-analyses showed that 25 genes, including cdc25a, in the cell cycle pathway were differentially expressed. Cdc25a mRNA levels in HCC tissues were higher than those in normal liver tissues in humans, rats and tree shrews, and the expression level of cdc25a in HCC tissues was higher than in corresponding paraneoplastic tissues in humans and rats. In human HCC tissues, the cdc25a mRNA level was significantly correlated with clinical stage, portal vein tumor thrombosis and extrahepatic metastasis. Western blotting showed that, cdc25a protein levels were significantly upregulated in HCC tissues in humans, rats and tree shrews. In conclusion, GSEA and meta-analysis can be combined to identify key molecules and pathways involved in HCC. This study demonstrated that the cell cycle pathway and the cdc25a gene may be crucial in the pathogenesis and progression of HCC.
\end{abstract}

Correspondence to: Professor Ji Cao, Department of Research, Affiliated Tumor Hospital of Guangxi Medical University, 71 Hedi Road, Nanning, Guangxi 530021, P.R. China

E-mail: caojicn@163.com

*Contributed equally

Key words: gene set enrich analysis, meta-analysis, cross-species, cdc25a, hepatocellular carcinoma

\section{Introduction}

Hepatocellular carcinoma (HCC) is one of the most common types of malignancy worldwide (1). It is associated with a poor prognosis in advanced stages. Identifying therapeutic targets for the treatment of HCC is important. During tumor development, somatically acquired "passenger" mutations owing to the inherent genomic instability of cancer cells, gene linkage, and spontaneous mutagenesis may not confer a selective advantage to the developing tumor (2). Currently, one of the challenges in cancer research is identifying key molecular changes among all acquired "passenger" mutations that promote the formation and development of the tumor. In recent years, genome sequencing projects have been completed in a number of animals, including humans, rats and mice, and genes have been identified that have the same expression in different species indicating that these genes could have conservative and important functions. The strategy of cross-species comparative oncogenomics was developed based on this understanding. Mattison et al (3) adopted cross-species comparative genomic hybridization to search for genes that were co-expressed in HCC tissues collected from humans, mice and rats, in order to identify novel candidate genes. The authors of the present study hypothesized that a search for genetic regulators common to humans and other animals, during HCC formation may aid in identifying key genes that affect the pathogenesis and progression of HCC. Gene microarrays have been widely used in HCC research. Analyses on whole-genome mRNA expression microarrays can aid in predicting transcripts that affect the prognosis and recurrence of HCC. However, identifying specific genetic markers that can be used as treatment targets remains a challenge. Mootha et al (4) developed GSEA, with which disease-associated gene pathways can be identified at the genomic level using case-control data. In GSEA, gene expression hybridization data in two biological conditions are analyzed to determine a pattern of gene expression in specific functional gene sets and whether such a pattern is statistically significant. In addition, due to differences in experimental platforms, samples, standardization methods and analytical methods, microarray data 
obtained in different laboratories may differ. Meta-analysis can be a viable solution to this problem, as it can be used to collect and quantitatively analyze data published on the same subject in an integrative manner, thus obtaining more accurate or a larger number of results than can be gained from any individual study (5). In the present study, GSEA and meta-analysis were combined to analyze whole genome and microarray data from five $\mathrm{HCC}$ data sets.

\section{Materials and methods}

Databases. A systematic literature and database search was performed to identify the HCC-related gene expression profiles of humans and other animals. Relevant data were downloaded from the Gene Expression Omnibus (GEO) database (http://www.ncbi.nlm.nih.gov/geo/). The keyword used for the search was 'hepatocellular carcinoma' and the research type was set as expression profiling by array. A total of 230 studies have published gene microarray data. The data sets that met the following criteria were included in this study: The data set must contain whole-genome mRNA expression microarray data; data included a comparison between HCC and normal tissues; both standardized and raw data sets were examined; and the data set had to include $>3$ samples. Using the above criteria, only five data sets (6-10) were included in the present study (Table I). GSEA and meta-analysis were combined to analyze whole genome and microarray data of these five HCC data sets. The genes that showed significantly differential expression were compared with the mRNA microarray results of a study conducted by our group using the tree shrew HCC model (11) to identify genes in HCC tissue that showed specific changes in $>2$ species (including humans).

\section{Sample collection.}

Human liver tissue samples. All patients have provided written informed consent to have their tissues stored and used for future research. The ethics committee of The Affiliated Tumor Hospital of Guangxi Medical University (Guangxi, China) approved this study. A total of 38 HCC tissue samples and corresponding paraneoplastic tissue samples ( $>2 \mathrm{~cm}$ away from the edge of the tumor) were obtained from patients (age, 24-70 years; mean age, 49.5 \pm 11.7 ) who underwent surgical resection at the Affiliated Tumor Hospital of Guangxi Medical University between January 2009 and December 2011. The diagnosis of HCC in each patient was confirmed histopathologically. Variables including tumor stage, presence of portal vein tumor thrombosis, number and size of tumors, serum $\alpha$-fetoprotein, tumor differentiation and presence of extrahepatic metastases and recurrence were evaluated in the patients with HCC. The tumor stage was determined according to the Barcelona-Clinic Liver Cancer staging classification (12). Tumor differentiation was graded according to the Edmondson grading system (12). Prior to surgical resection, none of the patients with $\mathrm{HCC}$ received chemotherapy. In addition, ten normal liver tissue samples surgically resected from patients with benign liver lesions were also collected.

Rat and tree shrew liver tissue samples. Animal experiments were conducted in accordance with the guidelines for The Care and Use of Laboratory Animals issued by the Ethics Committee of the Affiliated Tumor Hospital of Guangxi Medical
University. Rat and tree shrew animal models of HCC induced by aflatoxin B1 (AFB1) have been previously established by our group $(13,14)$. A total of 42 female Wistar rats (age, 4 weeks; weight, 180-210 g) were housed individually under controlled light (12 h light/dark cycle) and temperature conditions. Food and water were available ad libitum. The rats were randomly divided into two groups: An AFB1 group ( $n=28)$ and a control group ( $n=14)$. The rats in the AFB1 group were intraperitoneally injected with AFB1 (100-200 $\mu \mathrm{g} / \mathrm{kg}) 1-3$ times/week. Liver biopsies were obtained from all rats during the 14 th, 28 th, 42 nd and 55 th week, and all rats were sacrificed on the 64 th week by cervical dislocation. A total of 15 HCC tissue samples and 15 corresponding paraneoplastic tissues samples were collected from the rats in the AFB1 group. A total of 14 normal liver tissue samples were collected from the rats in the control group. Adult tree shrews $(n=27$; age, 6 months; weight, 100-160 g) were obtained from the Kunming Institute of Zoology, Chinese Science Academy (Yunnan, China), and were allowed to acclimatize for one week to the facilities prior to the experiment. The tree shrews were housed in individual, suspended, stainless steel wire cages, under controlled environmental conditions with a $12 \mathrm{~h}$ light/dark photoperiod. They had ad libitum access to tap water and a natural diet containing rice powder $(20 \%)$, corn powder $(20 \%)$, beef $(20 \%)$, wheat bran (10\%), soy bean (10\%), egg (10\%), whole milk powder $(5 \%)$, and a sugar, salt, vitamin, mineral mix (5\%). They were also fed daily with reconstituted powdered milk and fruit. The shrews were randomly divided into an AFB1 group ( $n=15 ; 9$ males and 6 females) and a control group ( $n=12 ; 6$ males and 6 females), and as previously reported (15), the animals in the AFB1 group were fed AFB1 (400 mg/kg body weight/day in a small amount of milk; Sigma-Aldrich, St. Louis, MO, USA) from the 1 st to the 90 th week of the experiment, whereas the animals in control group were raised without AFB1 administration. Liver biopsies from each animal were obtained every three months under Ketamine Hydrochloride anesthesia $(50 \mathrm{mg} / \mathrm{kg}$; Fujian Gutian Pharmaceutical Co., Ltd., Fujian, China). In the present study, $10 \mathrm{HCC}$ tissue samples and 10 corresponding paraneoplastic tissue samples were obtained when the animals developed cancer (HCC appeared at the 105 th week of experimentation) following sacrifice by cervical dislocation. A total of 10 normal liver tissue samples were collected from tree shrews in control group. After surgical resection, all tissue samples were subjected to rapid freezing in liquid nitrogen and then stored at $-80^{\circ} \mathrm{C}$. HCC samples were confirmed histopathologically.

GSEA and meta-analysis. Bioconductor (16) 2.10.1 was used to standardize the data. The Robust Multi-array Average (RMA) algorithm $(17,18)$ in the software package, Affy, was used for background correction, standardization and Log2 conversion of raw data on the Affymetrix platform. A t-test was performed to evaluate each probe in each data set. Only genes that were included in the Kyoto Encyclopedia of Genes and Genomes (KEGG) database (19) were subjected to GSEA. Genes with an interquartile range (IQR) $<0.5$ were excluded. If one gene corresponded to several probes, only the probe with the highest IQR was used. GSEA was performed using the Category package in Bioconductor. The gene sets represented by $\geq 10$ genes were subjected to further analyses, and genes in each pathway were subjected to a 
Table I. Basic information on the five whole-genome data sets.

\begin{tabular}{|c|c|c|c|c|c|c|c|}
\hline Data set & Authors, (ref.) & $\begin{array}{c}\text { Microarray } \\
\text { platform }\end{array}$ & $\begin{array}{l}\text { Experimental } \\
\text { design }\end{array}$ & $\begin{array}{l}\text { Number } \\
\text { of probes }\end{array}$ & Species & $\begin{array}{l}\text { Sample } \\
\text { (n) }\end{array}$ & $\begin{array}{c}\text { Control } \\
\text { (n) }\end{array}$ \\
\hline GSE19665 & Deng et al (6) & U133 Plus 2.0 & Paired tissues & 54000 & Homo sapiens & 10 & 10 \\
\hline GSE9809 & Liao et al (7) & Mouse430_2 & Unpaired+paired tissues & 45000 & Mus musculus & 3 & 7 \\
\hline GSE9012 & Khetchoumian et al (8) & Mouse430_2 & Unpaired tissues & 45000 & Mus musculus & 5 & 5 \\
\hline GSE19004 & Viatour et al (9) & Mouse430_2 & Unpaired tissues & 45000 & Mus musculus & 5 & 4 \\
\hline GSE2127 & Sheth et al (10) & moe430a & Paired tissues & 22000 & Mus musculus & 9 & 6 \\
\hline
\end{tabular}

Paired, control for the HCC group came from the same HCC individuals; unpaired, control for the HCC group came from normal individuals.

t-test. After 1,000 permutations, the P-value of each pathway was obtained. The upregulated and downregulated pathways in all five data sets were compared. The cell cycle pathway was upregulated in all five data sets. All genes in the cell cycle pathway were subjected to meta-analysis in each data set. T-tests were conducted using SAS9.13 software (Cary, $\mathrm{NC}$, USA) to calculate the P-value of each probe in the cell cycle pathway in each data set and the $\chi^{2}$ value of each gene was calculated using the following formula:

$$
\left(L S=\frac{\sum_{i=1}^{N}\left(-\log \left(\mathrm{p}_{i}\right)\right)}{N}\right)
$$

In which the degree of freedom is two times that of the data set $\mathrm{K})$. Genes with $\mathrm{P}<0.05$ were obtained. Analyses on these gene pathways were performed using the Database for Annotation, Visualization and Integrated Discover (DAVID; http://david. abcc.ncifcrf.gov/) in KEGG.

Reverse transcription-quantitative polymerase chain reaction (RT-qPCR). Total RNA was extracted from tissue samples using TRIzol reagent (Invitrogen; Thermo Fisher Scientific, Waltham, MA, USA), and first-strand cDNA was synthesized by reverse transcription from $1 \mu \mathrm{g}$ RNA using a RevertAid First Strand cDNA Synthesis kit (Thermo Fisher Scientific, Inc.). RT-qPCR was performed using a standard protocol from the SYBR ${ }^{\circledR}$ Premix Ex Taq kit (Takara, Dalian, China) on an Applied Biosystems 7300/7500 Real Time PCR system (Applied Biosystems, Grand Island, NY, USA). The cycling conditions were as follows: $95^{\circ} \mathrm{C}$ for $30 \mathrm{sec}$, and 40 cycles at $95^{\circ} \mathrm{C}$ for $5 \mathrm{sec}$ followed by $60^{\circ} \mathrm{C}$ for $34 \mathrm{sec}$. The relative mRNA expression levels of cdc $25 \mathrm{a}$ to control GAPDH were analyzed using ABI PRISM 7300 software v1.3.1 (Applied Biosystems) and calculated with the double standard curves method. The cdc 25 a forward and reverse primer sequences were 5'-CCAAAGGAACCATTGAGAAC-3' and 5'-CAGATGCCATAATTTCTGGAG-3', respectively, and the product length was $138 \mathrm{bp}$. The forward and reverse primer sequences for the internal control gene, 3-phosphate dehydrogenase (GAPDH), were 5'-AAGAAGGTGGTGAAGCAGGC-3' and 5'-ACCACCCTGTTGCTGTAGCC-3', respectively, and the product length was $200 \mathrm{bp}$.

Western blotanalysis. Western blot analysis to assess cdc25a and GAPDH expression was performed as previously described (14). Protein samples $(60 \mu \mathrm{g})$ were separated by $10 \%$ SDS-PAGE, prior to being transferred onto PVDF membranes (EMD Millipore, Billerica, MA, USA). The membranes were subsequently blocked with Tris-buffered saline with Tween 20 (TBST; Beyotime Institute of Biotechnology, Haimen, China) containing $5 \%(\mathrm{w} / \mathrm{v})$ skimmed milk powder for c. $2 \mathrm{~h}$ at room temperature and then incubated at $4^{\circ} \mathrm{C}$ overnight with the following primary antibodies: Rabbit polyclonal anti-human Cdc25 (cat. no. ab75743; Abcam, Cambridge, MA, USA) or mouse monoclonal anti-human glyceraldehyde 3-phosphate dehydrogenase (GAPDH; cat. no. TA-08; Beijing Zhongshan Golden Bridge Biotech Co., Ltd., Beijing, China) at dilutions of 1:100 or 1:1000, respectively. The membranes were then washed three times with TBST buffer, and incubated for $1 \mathrm{~h}$ at room temperature with IRDye 680LT goat anti-mouse secondary antibody (cat. no. 0926-68020; LI COR Biosciences, Lincoln, NE, USA) or IRDye 680LT goat anti-rabbit secondary antibody (cat. no. 926-68021; LI-COR Biosciences) at 1:7,500 dilution. The membranes were scanned using an Odyssey infrared imaging system (LI-COR Biosciences). Quantification of bands was achieved by ratiometric analysis of the fluorescent intensities of cdc25a and GAPDH using Odyssey Application Software 3.0 ( LI-COR Biosciences).

Statistical analysis. SPSS 13.0 (SPSS, Inc., Chicago, IL, USA) was used for data analysis. Quantitative data are expressed as the mean \pm standard deviation. Comparison of mean values between multiple groups was performed using a one-way analysis of variance. $\mathrm{P}<0.05$ was considered to indicate a statistically significant difference.

\section{Results}

Results of GSEA analysis. GSEA was performed for functional gene enrichment of the five data sets to search for key upregulated and downregulated pathways affecting these data sets. In GSE19665, 27 upregulated and 71 downregulated pathways were enriched; in GSE9809, 56 upregulated and 4 downregulated pathways were enriched; in GSE9012, 66 upregulated and 34 downregulated pathways were enriched; in GSE19004, 73 upregulated and 51 downregulated pathways were enriched; and in GSE2127, 69 upregulated and 50 downregulated pathways were enriched. There was a large amount of overlap between pathways in the GSE19665 and GSE19004 data sets. Downregulated pathways common to all five data sets included the linoleic acid metabolic pathway and the 
Table II. Distribution of differentially expressed genes revealed by meta-analyses of five data sets.

\begin{tabular}{ll}
\hline Gene & P-value \\
\hline ABI3BP & $3.23 \mathrm{E}-06$ \\
CCNB1 & $5.37 \mathrm{E}-06$ \\
NEK2 & $7.66 \mathrm{E}-06$ \\
MKI67 & $1.35 \mathrm{E}-05$ \\
cdc20 & $2.46 \mathrm{E}-05$ \\
angpt16 & $2.47 \mathrm{E}-05$ \\
rrm2 & $2.74 \mathrm{E}-05$ \\
Ttc36 & $3.06 \mathrm{E}-05$ \\
UBE2C & $3.18 \mathrm{E}-05$ \\
mcm2 & $4.07 \mathrm{E}-05$ \\
ASPM & $4.29 \mathrm{E}-05$ \\
NCAPH & $5.23 \mathrm{E}-05$ \\
TUBA1B & $5.23 \mathrm{E}-05$ \\
CCNB2 & $5.45 \mathrm{E}-05$ \\
Hist1h2ad & $5.71 \mathrm{E}-05$ \\
TOP2A & $5.91 \mathrm{E}-05$ \\
FOXM1 & $6.58 \mathrm{E}-05$ \\
BIRC5 & $7.18 \mathrm{E}-05$ \\
STMN1 & $7.74 \mathrm{E}-05$ \\
racgap1 & $7.84 \mathrm{E}-05$ \\
Hist1h2ag & $7.94 \mathrm{E}-05$ \\
Hist1h2ah & $7.94 \mathrm{E}-05$ \\
Hist1h2ai & $7.94 \mathrm{E}-05$ \\
CDCA5 & $9.89 \mathrm{E}-05$ \\
\hline & \\
\hline &
\end{tabular}

arachidonic acid metabolic pathway. Upregulated pathways common to all five data sets included the amino sugar and nucleotide sugar metabolic pathways, the cell cycle pathway and the thyroid cancer pathway.

Results of meta-analysis. A total of 220 positive genes were found in the GSE19665 data set and 213 positive genes were found in the remaining four data sets. After the P-value of each gene was obtained, SAS13.0 software was used for meta-analysis. A total of 1,708 genes were found to have $\mathrm{P}<0.05$ and 24 genes were found to have $\mathrm{P}<10^{-4}$ (Table II).

The 1,708 genes were subjected to pathway enrichment using the DAVID KEGG database. A total of 720 of these genes were found in the KEGG database.

Overlapping results obtained with GSEA and meta-analysis. The cell cycle pathway had the largest overlap between the GSEA and meta-analysis. Gene probe numbers in the cell cycle pathway of the five data sets were obtained using $\mathrm{R}$ programming language. The probe numbers were sent to the website, http://david.abcc.ncifcrf.gov/conversion.jsp, to obtain the official names of the genes. There were 99 differentially expressed genes in the cell cycle pathway of data set GSE19665, 96 in GSE9809, 90 in GSE9012, 106 in GSE19004 and 113 in GSE2127. Meta-analyses demonstrated that 25 genes involved in the cell cycle pathway were differentially
Table III. Distribution of differentially expressed genes in the cell cycle pathway.

\begin{tabular}{|c|c|c|}
\hline Gene & $\chi^{2}$ value & P-value \\
\hline $\mathrm{CCNB} 2$ & 46.94 & $9.67 \mathrm{E}-07$ \\
\hline $\mathrm{mcm} 2$ & 36.51 & $6.89 \mathrm{E}-05$ \\
\hline YWHAB & 35.63 & $9.74 \mathrm{E}-05$ \\
\hline CCNA2 & 33.83 & $2.00 \mathrm{E}-04$ \\
\hline CDKN2C & 32.60 & $3.00 \mathrm{E}-04$ \\
\hline Cdk1 & 32.24 & $4.00 \mathrm{E}-04$ \\
\hline MCM6 & 32.07 & $4.00 \mathrm{E}-04$ \\
\hline cdkn2b & 30.15 & $8.00 \mathrm{E}-04$ \\
\hline CCNB1 & 30.15 & $8.00 \mathrm{E}-04$ \\
\hline CDC25A & 29.98 & $9.00 \mathrm{E}-04$ \\
\hline $\operatorname{Mad} 211$ & 29.43 & $1.10 \mathrm{E}-03$ \\
\hline MCM7 & 28.57 & $1.50 \mathrm{E}-03$ \\
\hline CCNE1 & 28.21 & $1.70 \mathrm{E}-03$ \\
\hline MCM4 & 46.94 & $3.00 \mathrm{E}-03$ \\
\hline $\operatorname{cdc} 20$ & 36.51 & $6.50 \mathrm{E}-03$ \\
\hline smc3 & 35.63 & $6.70 \mathrm{E}-03$ \\
\hline penA & 33.83 & $7.90 \mathrm{E}-03$ \\
\hline RAD21 & 32.60 & $9.00 \mathrm{E}-03$ \\
\hline CDKN1A & 32.24 & $9.60 \mathrm{E}-03$ \\
\hline CCND1 & 32.07 & $1.08 \mathrm{E}-02$ \\
\hline SMAD3 & 30.15 & $1.16 \mathrm{E}-02$ \\
\hline TGFB1 & 30.15 & $1.27 \mathrm{E}-02$ \\
\hline YWHAZ & 29.98 & $2.06 \mathrm{E}-02$ \\
\hline YWHAG & 29.43 & $2.08 \mathrm{E}-02$ \\
\hline YWHAH & 28.57 & $2.52 \mathrm{E}-02$ \\
\hline
\end{tabular}

expressed $(\mathrm{P}<0.05)$. The names, $\chi^{2}$ values and $\mathrm{P}$-values of these genes are shown in Table III.

Cdc25a mRNA expression. The results of the present study identified 25 differential expression genes in the cell cycle signaling pathway, as determined by GSEA and meta-analysis which analyzed five data sets from human HCC tissue samples. Among the 25 candidate genes, Cdc25a was also shown to be overexpressed in the HCC tissue samples of diethylnitrosamine-induced rats and aflatoxin B1-induced tree shrews $(11,20)$. Therefore, Cdc25a mRNA expression was further investigated. Results of real-time fluorescent PCR showed that the cdc25a mRNA expression level in human HCC tissue $(0.00425 \pm 0.00241)$ was significantly higher than in the corresponding paraneoplastic tissue $(0.00086 \pm 0.00081)$ $(\mathrm{P}<0.05)$ and in normal liver tissue $(0.00038 \pm 0.00032)$ $(\mathrm{P}<0.05)$. There was no significant difference between the paraneoplastic tissue and normal liver tissue $(\mathrm{P}>0.05)$. The cdc25a mRNA expression level in rat HCC tissue $(0.00281 \pm 0.00278)$ was significantly higher than that in the corresponding paraneoplastic tissue $(0.00044 \pm 0.00035)$ $(\mathrm{P}<0.05)$ and in normal liver tissue $(0.00051 \pm 0.00022)$ $(\mathrm{P}<0.05)$; however, there was no significant difference between the paraneoplastic tissue and normal liver tissue $(\mathrm{P}>0.05)$. The cdc25a mRNA expression level in tree shrew HCC 
Table IV. Correlation between the detection rate of cdc25a mRNA in human HCC tissues and clinical parameters.

\begin{tabular}{|c|c|c|c|c|}
\hline Clinical parameter & $\mathrm{n}$ & Cdc25a mRNA expression level & t-value & P-value \\
\hline \multicolumn{5}{|l|}{ BCLC stage (12) } \\
\hline $0, \mathrm{~A}$ & 26 & $0.00253 \pm 0.00175$ & 2.342 & 0.023 \\
\hline $\mathrm{B}, \mathrm{C}$ & 12 & $0.00537 \pm 0.00221$ & & \\
\hline \multicolumn{5}{|l|}{ PVTT } \\
\hline Yes & 10 & $0.00564 \pm 0.00259$ & 0.505 & 0.030 \\
\hline No & 28 & $0.00376 \pm 0.00214$ & & \\
\hline \multicolumn{5}{|l|}{ Extrahepatic metastasis } \\
\hline Yes & 11 & $0.00571 \pm 0.00254$ & 0.139 & 0.014 \\
\hline No & 27 & $0.00367 \pm 0.00208$ & & \\
\hline \multicolumn{5}{|l|}{ Recurrence } \\
\hline Yes & 20 & $0.00408 \pm 0.00250$ & 0.071 & 0.632 \\
\hline No & 18 & $0.00446 \pm 0.00229$ & & \\
\hline \multicolumn{5}{|l|}{ Tumor diameter $(\mathrm{cm})$} \\
\hline$\geq 5$ & 31 & $0.00436 \pm 0.00236$ & 0.218 & 0.568 \\
\hline$<5$ & 7 & $0.00378 \pm 0.00262$ & & \\
\hline \multicolumn{5}{|l|}{ Number of tumors } \\
\hline 1 & 24 & $0.00375 \pm 0.00203$ & 1.886 & 0.083 \\
\hline$\geq 2$ & 14 & $0.00513 \pm 0.00274$ & & \\
\hline \multicolumn{5}{|l|}{ Serum AFP $(\mu \mathrm{g} / \mathrm{l})$} \\
\hline$\geq 400$ & 13 & $0.00521 \pm 0.00249$ & 0.215 & 0.075 \\
\hline$<400$ & 25 & $0.00376 \pm 0.00221$ & & \\
\hline \multicolumn{5}{|l|}{ Tumor differentiation } \\
\hline Highly differentiated & 21 & $0.00434 \pm 0.00217$ & 1.602 & 0.825 \\
\hline Poorly differentiated and undifferentiated & 17 & $0.00416 \pm 0.00268$ & & \\
\hline
\end{tabular}

BCLC, Barcelona Clinic Liver Cancer; PVTT, portal vein tumor thrombus; AFP, $\alpha$-fetoprotein.

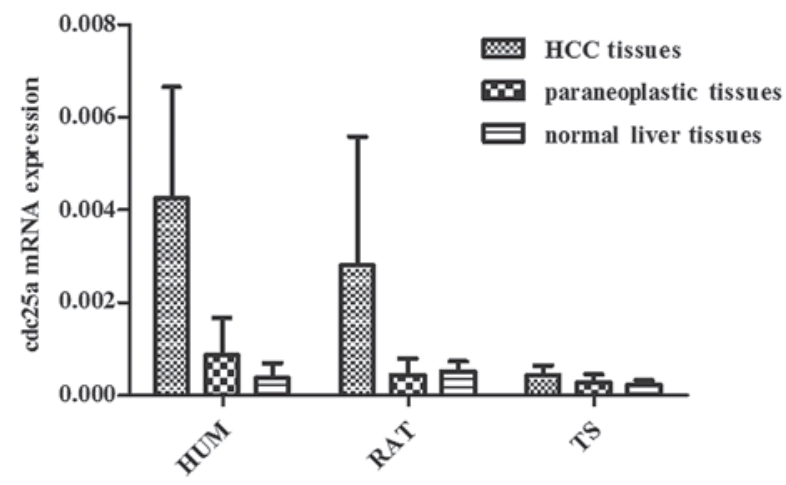

Figure 1. Expression of cdc25a mRNA in the HCC, paraneoplastic and normal liver tissues of different species (humans, rats and tree shrews). HUM, human; RAT, rat; TS, tree shrew.

tissue $(0.00043 \pm 0.00021)$ was significantly higher than that in normal liver tissue $(0.00022 \pm 0.00010)(\mathrm{P}<0.05)$, however there was no significant difference between the paraneoplastic tissue $(0.00028 \pm 0.00017)$ and corresponding HCC tissue or normal liver tissue ( $\mathrm{P}>0.05$; Fig. 1$)$.

The 38 HCC patients were grouped on the basis of whether they had PVTT, extrahepatic metastasis, BCLC stage,

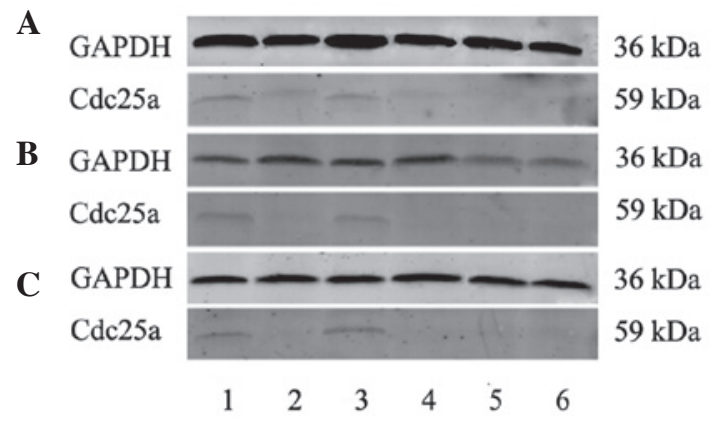

Figure 2. Cdc25a protein expression in the HCC, paraneoplastic and normal liver tissues of different species (humans, rats and tree shrews), revealed by western blot analysis. (A) cdc25a protein expression in human liver tissues; (B) cdc25a protein expression in rat liver tissues; and $(\mathrm{C})$ cdc25a protein expression in tree shrew liver tissues. Lanes 1 and 3, HCC tissue; lanes 2 and 4, corresponding paraneoplastic tissue; and lanes 5 and 6, normal liver tissue. GAPDH, 3-glyceraldehyde phosphate dehydrogenase.

postoperative recurrence, tumor diameter, number of tumors, serum AFP level and degree of tumor differentiation. The correlation between cdc25a mRNA expression in the HCC tissues and the above clinical parameters was examined. As demonstrated in Table IV, the detection rate of cdc25a mRNA 
was observed to be significantly correlated with Barcelona Clinic Liver Cancer (BCLC) stage, portal vein tumor thrombus (PVTT) and extrahepatic metastasis, but not with postoperative recurrence, tumor diameter, tumor number, serum $\alpha$-fetoprotein (AFP) level or degree of tumor differentiation.

Cdc25a protein expression. Based on the results of fluorescent quantitative PCR, 38 human HCC and corresponding paraneoplastic tissue samples, 10 normal human liver tissue samples, 15 rat $\mathrm{HCC}$ and corresponding paraneoplastic tissue samples, 10 normal rat liver tissues, 10 tree shrew HCC and corresponding paraneoplastic tissue samples, and 10 normal tree shrew liver tissue samples were collected for western blot analysis. Specific cdc25a and GAPDH protein bands were observed at 36 and $59 \mathrm{kDa}$ of pre-stained proteins, respectively, although the target protein, cdc25a, was not detected in normal human liver tissue, or in the normal or paraneoplastic liver tissues of rats or tree shrews. The mean relative cdc25a expression level in human HCC tissues $(0.339 \pm 0.239)$ was significantly higher than in the corresponding paraneoplastic tissues $(0.0609 \pm 0.0498 ; \mathrm{P}<0.05)$. In humans, rats and tree shrews, the cdc 25 a bands were notably stronger in HCC tissues than in the corresponding paraneoplastic and normal liver tissues, consistent with the high cdc25a mRNA expression in HCC tissues (Fig. 2).

\section{Discussion}

Analyses on gene microarray data are an important part of gene microarray research. A lot of useful information may be missed due to potential problems with the samples when analyzing the results of a single experiment. In addition, using a t-test to target a single set of microarray data has certain limitations. The small sample size may lead to an unreliable variance estimation, resulting in a high false-positive rate, whereas differences in the expression levels of different samples may be ignored (21). In GSEA, microarray data of samples in two different biological states (e.g., normal vs. cancerous) are analyzed to determine whether a set of genes show similar expression trends between the two states, thereby identifying genes or pathways associated with the disease (22).

In the present study, GSEA and meta-analysis were combined to analyze five data sets, and the two results were compared in order to identify HCC-related genes and pathways. DEGs in $>2$ groups of samples were analyzed using GSEA and the clustering was performed using differentially expressed genes. $\mathrm{R}$ programming language was used to process the data and for statistical analysis to obtain pathways that showed changes common to all five data sets. DEGs were analyzed using the DAVID website to determine the pathways in which these genes may be involved. The cell cycle pathway statistically significant in the results obtained with the GSEA and meta-analysis and was further analyzed to identify genes with significant differential expression.

Among the 25 differentially expressed genes in the cell cycle pathway that were identified, cyclin B2, cyclin B1, cyclin D1, cdc25a and cdk1 are closely associated with HCC occurrence and development (23-26). Cyclin-dependent kinases $(\mathrm{CDKs})$ are core proteins in the cell cycle regulatory network. Changes in the expression activity of CDKs directly affect the length of the cell cycle, determine the progression of the cell cycle, and are closely associated with the growth, differentiation, migration and apoptosis of normal cells, as well as the occurrence, development and metastasis of tumors (27). CDKs are important in the regulation of hepatoma cell proliferation and apoptosis. Cyclin B and cyclin D are members of the cyclin family and determine which CDKs phosphorylate which substrates as well as when and where. Studies have shown that (27) Cyclin D1 is expressed in normal liver tissue, but its expression is increased in HCC tissue, and is correlated with the histological grade of HCC. This suggests that Cyclin D1 is involved in the progression and development of HCC and may promote cell proliferation, contributing to tumor formation. Cyclin B1 is in the same family, is highly expressed in HCC tissue and is key in the transition process of $\mathrm{HCC}$ cells. Cdc25A is a phosphatase with dual specificity, and can activate CDKs, promoting the progression of the cell cycle.

Studies have shown that cdc25a is associated with breast cancer (28), non-small cell lung cancer (29), colorectal cancer (30), prostate cancer (31) and other malignant tumors. However, there are few studies regarding the correlation between cdc25a and liver cancer. In a study by Xu et al (26), cdc25a expression was examined in HCC tissues using RT-PCR, immunohistochemistry and western blotting, and reported cdc25a expression detection rates of 69 (9/13), 56 (33/59) and 78\% (46/59), respectively. Immunohistochemistry tests showed that high cdc25a protein expression was positively correlated with poor tumor differentiation and PVTT. In the present study, fluorescent quantitative PCR and western blotting showed that cdc25a expression was high in human HCC tissues at the transcriptional and at the translational levels. The cdc25a mRNA expression level in human HCC tissues was found to be positively correlated with PVTT and extrahepatic metastasis. This suggests that cdc25a is associated with the progression of $\mathrm{HCC}$, and may serve as an indicator to evaluate the severity of the disease. The differences between the results of the present study and those of Xu et al (26) may be due to differences in sample sources, transcription and translation levels, and experimental method.

In this study, overexpression of cdc25a was also found in HCC samples from aflatoxin B1-induced rat and tree shrew HCC models. Such consistent differential expression in different species has also been reported in a previous study by our group (11). The same cross-species expression patterns suggest that cdc25a is key in the pathogenesis and progression of HCC. Xu et al (32) explored the possibility of using cdc25a as an antitumor target by inhibiting its activity in HCC cell lines. It was reported that antisense oligonucleotides of cdc25a inhibited $25-50 \%$ of cell growth within $48 \mathrm{~h}$, resulting in arrest at the G0-G1 phase and effectively suppressing the proliferation of HCC cells. This suggests that cdc25a can serve as a feasible target for anticancer treatment. In addition, a study by Liu et al (33) showed that knockdown of the ROCK2 gene could activate the reduced ubiquitin-proteasome pathway, thereby promoting cdc25a ubiquitination, which would ultimately lead to cdc25a degradation and inhibition of HCC cell growth. Therefore, cdc25a may be a novel target for anti-HCC treatment.

In conclusion, GSEA and meta-analysis can be combined to identify key molecules and pathways involved in the 
pathogenesis and progression of HCC. The cell cycle pathway and the cdc25a gene may be the crucial in the pathogenesis and progression of HCC.

\section{Acknowledgements}

The present study was supported by the National Science Foundation of China (grant no. 30960428).

\section{References}

1. Waly Raphael S, Yangde Z and Yuxiang C: Hepatocellular carcinoma: Focus on different aspects of management. ISRN Oncol 2012: 421673, 2012.

2. Beroukhim R, Mermel CH, Porter D, Wei G, Raychaudhuri S, Donovan J, Barretina J, Boehm JS, Dobson J, Urashima M, et al The landscape of somatic copy-number alteration across human cancers. Nature 463: 899-905, 2010.

3. Mattison J, Kool J, Uren AG, de Ridder J, Wessels L, Jonkers J, Bignell GR, Butler A, Rust AG, Brosch M, et al: Novel candidate cancer genes identified by a large-scale cross-species comparative oncogenomics approach. Cancer Res 70: 883-895, 2010.

4. Mootha VK, Lindgren CM, Eriksson KF, Subramanian A, Sihag S, Lehar J, Puigserver P, Carlsson E, Ridderstråle M, Laurila E, et al: PGC-1alpha-responsive genes involved in oxidative phosphorylation are coordinately downregulated in human diabetes. Nat Genet 34: 267-273, 2003.

5. Greenbaum D, Jansen R and Gerstein M: Analysis of mRNA expression and protein abundance data: An approach for the comparison of the enrichment of features in the cellular population of proteins and transcripts. Bioinformatics 18: 585-596, 2002.

6. Deng YB, Nagae G, Midorikawa Y, Yagi K, Tsutsumi S, Yamamoto S, Hasegawa K, Kokudo N, Aburatani H and Kaneda A: Identification of genes preferentially methylated in hepatitis $\mathrm{C}$ virus-related hepatocellular carcinoma. Cancer Sci 101: 1501-1510, 2010.

7. Liao YJ, Liu SP, Lee CM, Yen CH, Chuang PC, Chen CY, Tsai TF, Huang SF, Lee YH and Chen YM: Characterization of a glycine $\mathrm{N}$-methyltransferase gene knockout mouse model for hepatocellular carcinoma: Implications of the gender disparity in liver cancer susceptibility. Int J Cancer 124: 816-826, 2009.

8. Khetchoumian K, Teletin M, Tisserand J, Mark M, Herquel B, Ignat M, Zucman-Rossi J, Cammas F, Lerouge T, Thibault C, et al: Loss of Trim 24 (Tiflalpha) gene function confers oncogenic activity to retinoic acid receptor alpha. Nat Genet 39: 1500-1506, 2007.

9. Viatour P and Sage J: Mouse HCC model. http://www.ncbi.nlm. nih.gov/geo/query/acc.cgi?acc=GSE19004. Accessed Jul 28, 2012.

10. Sheth SS, Bodnar JS, Ghazalpour A, Thipphavong CK, Tsutsumi S, Tward AD, Demant P, Kodama T, Aburatani $\mathrm{H}$ and Lusis AJ: Hepatocellular carcinoma in Txnip-deficient mice. Oncogene 25: 3528-3536, 2006.

11. Li Y, Wan DF, Su JJ, Cao J, Ou C, Qiu XK, Ban KC, Yang C, Qin LL, Luo D, et al: Differential expression of genes during aflatoxin B(1)-induced hepatocarcinogenesis in tree shrews. World J Gastroenterol 10: 497-504, 2004.

12. Sobin LH and Compton CC:TNM seventh edition: what's new, what's changed: communication from the International Union Against Cancer and the American Joint Committee on Cancer. Cancer 116:5336-5339, 2010

13. Li Y, Qin X, Cui J, Dai Z, Kang X, Yue H, Zhang Y, Su J, Cao J, Ou C, et al: Proteome analysis of aflatoxin B1-induced hepatocarcinogenesis in tree shrew (Tupaia belangeri chinensis) and functional identification of candidate protein peroxiredoxin II. Proteomics 8: 1490-1501, 2008.

14. Hao YR, Yang F, Cao J, Ou C, Zhang JJ, Yang C, Duan XX, Li Y and Su JJ: Ginkgo biloba extracts (EGb761) inhibits aflatoxin B1-induced hepatocarcinogenesis in Wistar rats. Zhong Yao Cai 32: 92-96, 2009 (In Chinese).
15. Li Y, Su JJ, Qin LL, Egner PA, Wang J, Groopman JD, Kensler TW and Roebuck BD: Reduction of aflatoxin B1 adduct biomarkers by oltipraz in the tree shrew (Tupaia belangeri chinensis). Cancer Lett 154: 79-83, 2000

16. Gentleman RC, Carey VJ, Bates DM, Bolstad B, Dettling M, Dudoit S, Ellis B, Gautier L, Ge Y, Gentry J, et al: Bioconductor: Open software development for computational biology and bioinformatics. Genome Biol 5: R80, 2004

17. Irizarry RA, Hobbs B, Collin F, Beazer-Barclay YD, Antonellis KJ, Scherf U and Speed TP: Exploration, normalization, and summaries of high density oligonucleotide array probe level data. Biostatistics 4: 249-264, 2003.

18. Gautier L, Cope L, Bolstad BM and Irizarry RA: Affy-analysis of affymetrix GeneChip data at the probe level. Bioinformatics 20: 307-315, 2004.

19. Kanehisa M and Goto S: KEGG: Kyoto encyclopedia of genes and genomes. Nucleic Acids Res 28: 27-30, 2000.

20. Liang HJ, Wei W, Kang XN, Guo K, Cao J, Su JJ, Yang C, Ou C, Li Y and Liu YK: Differentially expressed proteins in the precancerous stage of rat hepatocarcinogenesis induced by diethylnitrosamine. Chinese Journal of Hepatology 17: 669-674, 2009 (In Chinese)

21. MacDonald JW and Ghosh D: COPA-cancer outlier profile analysis. Bioinformatics 22: 2950-2951, 2006.

22. Subramanian A, Kuehn H, Gould J, Tamayo P and Mesirov JP GSEA-P: A desktop application for gene set enrichment analysis. Bioinformatics 23: 3251-3253, 2007.

23. Takashima S, Saito H, Takahashi N, Imai K, Kudo S, Atari M, Saito Y, Motoyama S and Minamiya Y: Strong expression of cyclin B2 mRNA correlates with a poor prognosis in patients with non-small cell lung cancer. Tumour Biol 35: 4257-4265, 2014

24. Gao SY, Li J, Qu XY, Zhu N and Ji YB: Downregulation of Cdk1 and cyclinB1 expression contributes to oridonin-induced cell cycle arrest at G2/M phase and growth inhibition in SGC-7901 gastric cancer cells. Asian Pac J Cancer Prev 15: 6437-6441, 2014.

25. Li T, Zhao X, Mo Z, Huang W, Yan H, Ling Z and Ye Y: Formononetin promotes cell cycle arrest via downregulation of Akt/Cyclin D1/CDK4 in human prostate cancer cells. Cell Physiol Biochem 34: 1351-1358, 2014.

26. Xu X, Yamamoto H, Sakon M, Yasui M, Ngan CY, Fukunaga H, Morita T, Ogawa M, Nagano H, Nakamori S, et al: Overexpression of CDC25A phosphatase is associated with hypergrowth activity and poor prognosis of human hepatocellular carcinomas. Clin Cancer Res 9: 1764-1772, 2003.

27. Liu L, Schwartz B, Tsubota Y, Raines E, Kiyokawa H, Yonekawa K, Harlan JM and Schnapp LM: Cyclin-dependent kinase inhibitors block leukocyte adhesion and migration. J Immunol 180: 1808-1817, 2008.

28. Brunetto E, Ferrara AM, Rampoldi F, Talarico A, Cin ED, Grassini G, Spagnuolo L, Sassi I, Ferro A, Cuorvo LV, et al: CDC25A protein stability represents a previously unrecognized target of HER2 signaling in human breast cancer: Implication for a potential clinical relevance in trastuzumab treatment. Neoplasia 15: 579-590, 2013.

29. Younis RH, Cao W, Lin R, Xia R, Liu Z, Edelman MJ, Mei Y, Mao L and Ren H: CDC25A (Q110del): A novel cell division cycle $25 \mathrm{~A}$ isoform aberrantly expressed in non-small cell lung cancer. PLoS One 7: e46464, 2012.

30. Huang MY, Wang JY, Chang HJ, Kuo CW, Tok TS and Lin SR. CDC25A, VAV1, TP73, BRCA1 and ZAP70 gene overexpression correlates with radiation response in colorectal cancer. Oncol Rep 25: 1297-1306, 2011.

31. Chiu YT, Han HY, Leung SC, Yuen HF, Chau CW, Guo Z, Qiu Y, Chan KW, Wang X, Wong YC and Ling MT: CDC25A functions as a novel Ar corepressor in prostate cancer cells. J Mol Biol 385: 446-456, 2009.

32. Xu X, Yamamoto H, Liu G, Ito Y, Ngan CY, Kondo M, Nagano H, Dono K, Sekimoto M and Monden M: CDC25A inhibition suppresses the growth and invasion of human hepatocellular carcinoma cells. Int J Mol Med 21: 145-152, 2008.

33. Liu T, Yu X, Li G, Yuan R, Wang Q, Tang P, Wu L, Liu X, Peng $\mathrm{X}$ and Shao J: Rock2 regulates Cdc25A through ubiquitin proteasome system in hepatocellular carcinoma cells. Exp Cell Res 318: 1994-2003, 2012. 